\title{
Criminologie
}

\section{La coévolution du « vol d'identité » et des systèmes de paiement}

\section{Benoit Dupont}

Volume 43, numéro 2, automne 2010

Les 50 ans de l'École de criminologie : aperçu de la recherche d'ici et d'ailleurs

URI : https://id.erudit.org/iderudit/1001777ar

DOI : https://doi.org/10.7202/1001777ar

Aller au sommaire du numéro

\section{Éditeur(s)}

Les Presses de l’Université de Montréal

ISSN

0316-0041 (imprimé)

1492-1367 (numérique)

Découvrir la revue

Citer cet article

Dupont, B. (2010). La coévolution du « vol d'identité » et des systèmes de paiement. Criminologie, 43(2), 247-268. https://doi.org/10.7202/1001777ar
Résumé de l'article

Dans un article devenu un classique de la criminologie, Edwin Sutherland soulignait dès 1940 les bénéfices réciproques que pourraient tirer les économistes et les criminologues d'une meilleure intégration de leurs connaissances. Bien que son invitation à l'interdisciplinarité porte avant tout sur le crime en col blanc, d'autres formes de délinquance se prêtent également particulièrement bien à une telle démarche, au premier rang desquelles figure le " vol d'identité ". Après avoir examiné dans la première partie de cet article les ambiguïtés et les paradoxes qui pèsent sur les analyses contemporaines du " vol d'identité ", je propose dans la seconde partie un cadre d'analyse alternatif qui repose sur le concept de coévolution et liant le " vol d'identité " tel que nous le connaissons aujourd'hui au développement de nouveaux moyens de paiement à partir de la fin des années 1950. À l'aide de données historiques et économiques, je montre comment cette transformation fondamentale des habitudes de consommation a contribué à l'émergence de nouvelles opportunités criminelles, au développement de mécanismes de sécurité et de procédures privées de règlement des conflits, ainsi qu’au déclin d'autres formes de délinquance.
Ce document est protégé par la loi sur le droit d'auteur. L'utilisation des services d’Érudit (y compris la reproduction) est assujettie à sa politique d'utilisation que vous pouvez consulter en ligne.

https://apropos.erudit.org/fr/usagers/politique-dutilisation/ 


\title{
La coévolution du «vol d'identité » et des systèmes de paiement
}

\author{
Benoit Dupont \\ Directeur \\ Centre international de criminologie comparée (CICC) \\ Titulaire de la Chaire de recherche du Canada en sécurité, identité et technologie \\ benoit.dupont@umontreal.ca
}

RÉSUMÉ - Dans un article devenu un classique de la criminologie, Edwin Sutherland soulignait dès 1940 les bénéfices réciproques que pourraient tirer les économistes et les criminologues d'une meilleure intégration de leurs connaissances. Bien que son invitation à l'interdisciplinarité porte avant tout sur le crime en col blanc, d'autres formes de délinquance se prêtent également particulièrement bien à une telle démarche, au premier rang desquelles figure le "vol d'identité». Après avoir examiné dans la première partie de cet article les ambiguïtés et les paradoxes qui pèsent sur les analyses contemporaines $d u$ "vol d'identité», je propose dans la seconde partie un cadre d'analyse alternatif qui repose sur le concept de coévolution et liant le «vol d'identité» tel que nous le connaissons aujourd'hui au développement de nouveaux moyens de paiement à partir de la fin des années 1950. À l'aide de données historiques et économiques, je montre comment cette transformation fondamentale des habitudes de consommation a contribué à l'émergence de nouvelles opportunités criminelles, au développement de mécanismes de sécurité et de procédures privées de règlement des conflits, ainsi qu'au déclin d'autres formes de délinquance.

MOTS-CLÉS - Vol d'identité, moyens de paiement, cartes de crédit, coévolution, opportunités criminelles, procédures privées de règlement des conflits.

\section{Introduction}

Dans un article devenu un classique de la criminologie, Edwin Sutherland soulignait dès 1940 les bénéfices réciproques que pourraient tirer les économistes et les criminologues d'une meilleure intégration de leurs connaissances. Il aurait sans conteste pu associer les historiens à cette alliance intellectuelle destinée à forger une théorie criminologique moins inféodée aux stéréotypes populaires qui tendent à simplifier les 
causes de la délinquance et les moyens de la prévenir. Bien que son invitation à l'interdisciplinarité porte avant tout sur le crime en col blanc, d'autres formes de délinquance se prêtent également particulièrement bien à une telle démarche, au premier rang desquelles figure le «vol d'identité».

Alors que le «vol d'identité» est fréquemment désigné comme le crime connaissant la plus forte croissance depuis quelques années (Finklea, 2009), son traitement criminologique demeure très superficiel: tout d'abord, la pertinence de la terminologie utilisée est loin de faire l'unanimité, ce qui explique pourquoi il serait préférable de placer ce terme entre guillemets dans un article scientifique, même si une telle approche alourdit le style rédactionnel. Ensuite, les sondages de victimisation cherchant à mesurer l'étendue du problème sont majoritairement commandités par des fournisseurs de produits ou de services de sécurité dont les intérêts particuliers biaisent nécessairement les méthodologies et les analyses. Enfin, l'activité répressive de l'État étant réduite à sa plus simple expression dans ce domaine, la source traditionnelle de connaissances des criminologues (les individus incarcérés dans les établissements correctionnels) est quasiment absente, ce qui limite le nombre de recherches sur les auteurs de ce type de fraude et sur leurs méthodes.

Le déficit de connaissances qui découle de ces trois facteurs fait notamment sentir ses effets de deux manières: tout d'abord, on observe une propension des médias à entretenir un discours alarmiste quant à la gravité du problème et à ses manifestations. Par un processus de distorsion repéré par Stanley Cohen (1972), les médias tendent à se focaliser sur les affaires les plus édifiantes de «vol d'identité», et tout particulièrement sur celles qui font ressortir une asymétrie entre la vulnérabilité et l'impuissance des victimes, d'une part, et l'ingéniosité et l'expertise des délinquants, d'autre part. Dans un tel contexte, les conseils prodigués par les divers «experts» mobilisés pour expliquer aux consommateurs comment se protéger s'appuient sur des connaissances anecdotiques qui reflètent rarement la structure des risques objectifs. On dissuade par exemple les internautes de divulguer des informations personnelles sur les sites de socialisation en ligne comme Facebook, alors que cette source d'identifiants n'est exploitée que de manière marginale par les délinquants. Il existe d'excellentes raisons d'exercer un certain discernement dans le dévoilement de sa vie privée, mais la lutte contre le «vol d'identité» n'en fait certainement pas partie. 
Face à de telles limites, il est tentant de discréditer le «vol d'identité» comme un effet de mode qui exploite l'inquiétude de l'opinion publique face à l'émergence de risques inhérents au consumérisme dominant des sociétés modernes avancées (Marron, 2008; Monahan, 2009). Si cette posture critique possède l'immense avantage de chercher à replacer le «vol d'identité» dans un contexte économique et social plus large, elle court néanmoins le risque de s'effondrer sous le poids de ses propres stéréotypes lorsqu'elle invoque des arguments préformatés tels que les inégalités sociales, la flexibilité excessive imposée aux employés sousqualifiés, la stigmatisation des consommateurs de produits stupéfiants ou encore le déploiement de modes panoptiques de gouvernance des conduites individuelles pour en analyser les causes et les effets (Monahan, 2009: 169). Une approche intermédiaire est toutefois possible, qui évite à la fois de céder à un alarmisme a-théorique et de sombrer dans un scepticisme réfractaire à tout empirisme. Celle-ci associe l'étude du «vol d'identité » à l'évolution des systèmes de paiement de ces soixante dernières années, et plus particulièrement à la généralisation des cartes de crédit et de débit comme moyen privilégié de règlement. En analysant les conditions historiques et économiques liées à la dématérialisation des moyens de paiement, il devient possible de comprendre certains des paradoxes liés au "vol d'identité», et notamment les raisons pour lesquelles l'État manifeste un intérêt très relatif pour le phénomène malgré le matraquage médiatique dont ce dernier fait l'objet, ou encore pourquoi la légitimité des institutions financières ne semble pas affectée, en dépit des préjudices financiers considérables auxquels ces dernières - ainsi que leurs clients - sont exposés.

Dans la première partie de cet article, j'examine ainsi les ambiguités et les paradoxes qui pèsent sur les analyses contemporaines du «vol d'identité». L'imprécision du terme, le manque de fiabilité des statistiques recueillies afin d'en mesurer l'ampleur, l'absence de connaissances concernant les délinquants qui en sont les auteurs et le maintien de la confiance des consommateurs aux institutions financières qui y sont le plus exposées seront successivement abordés. Dans une seconde partie, je propose un cadre d'analyse alternatif qui repose sur le concept de coévolution et liant le «vol d'identité» tel que nous le connaissons aujourd'hui au développement de nouveaux moyens de paiement à partir de la fin des années 1950. Je montre comment cette transformation fondamentale des habitudes de consommation a contribué à 
l'émergence de nouvelles opportunités criminelles, au développement de mécanismes de sécurité et de procédures privées de règlement des conflits, ainsi qu'au déclin d'autres formes de délinquance.

\section{Le « vol d'identité »: un crime paradoxal}

Le terme «vol d'identité» fit son apparition au milieu des années 1990, lorsque des associations américaines et canadiennes de défense des consommateurs et de protection de la vie privée s'émurent de la multiplication des cas de fraude reposant sur l'acquisition abusive et la manipulation des données personnelles des victimes, et de la difficulté qu'avaient ces dernières à se faire reconnaître comme telles par les institutions policières, judiciaires et financières (Cavoukian, 1997; Newman et McNally, 2005 : 2). L'existence légale du vol d'identité fut consacrée pour la première fois aux États-Unis en 1998 par le vote d'une loi fédérale (l'Identity Theft and Assumption Deterrence Act) permettant de criminaliser le transfert ou l'usage d'informations personnelles à des fins illégales (Saunders et Zucker, 1999; Pontell, 2009: 264). Au Canada, il fallut attendre octobre 2009 pour qu'une loi vienne amender le Code criminel afin d'y inclure l'infraction de vol d'identité (art. 402.2), destinée à réprimer les étapes préparatoires (obtention, possession, transfert ou vente de renseignements identificateurs) de ce crime. Cependant, si une telle activité législative a considérablement facilité le travail des policiers et des procureurs, les chercheurs restent confrontés à la difficulté apparemment insoluble de définir de manière cohérente une multitude de pratiques délinquantes très hétérogènes (Newman et McNally, 2005 : iv), dont certaines ont pu être analysées dès les années 1980 sans jamais faire référence à cette terminologie (Tremblay, 1986).

\section{Une terminologie contestable}

Le terme «vol d'identité» est en effet particulièrement mal choisi pour désigner les activités frauduleuses complexes qui lui sont associées. Le mot «vol» induit d'abord en erreur, puisqu'il implique l'impossibilité pour la victime de jouir du bien dérobé (assimilable au résultat d'un jeu à somme nulle), alors que le «vol d'identité» se caractérise au contraire par l'exploitation simultanée des éléments identificateurs de la victime par cette dernière et par le délinquant (Koops et al., 2009: 11). Autrement dit, les conséquences négatives de l'usage illégal des identi- 
fiants de la victime ne privent pas cette dernière de son identité. Tout au plus lui font-elles perdre de manière momentanée la jouissance de certains avantages instrumentaux comme un score de crédit élevé et la capacité d'emprunter de l'argent aux banques. Il serait donc plus juste de faire référence à un «emprunt d'identité» (Jones et Levi, 2000: 1) ou à une «manipulation d'identité», le vol ne concernant à proprement parler que des documents identificateurs pouvant être facilement remplacés, des informations personnelles permettant l'accès à divers services, et dans certains cas, des avoirs financiers confiés à des institutions bancaires.

De surcroît, la déclinaison du mot «identité» au singulier laisse entendre que celle-ci constitue un ensemble homogène et cohérent de caractéristiques qui établissent l'individualité. Or, l'identité d'une personne se compose plutôt d'un agrégat disparate d'éléments identificateurs attribués (un numéro d'assurance sociale), biométriques (des empreintes digitales), biographiques (le statut matrimonial) ou même choisis (un identifiant et un mot de passe pour accéder à un compte en ligne) (Jones et Levi, 2000: 3 ; Koops et al., 2009: 4) qui disposent d'un potentiel très variable d'exploitation par les fraudeurs. Si l'on souhaite conserver le terme de «vol», celui-ci doit donc faire référence aux éléments identificateurs dans toute leur diversité plutôt qu'à une identité singulière.

Devant une telle imprécision terminologique, il serait plus profitable de désigner l'enchaînement des phases d'acquisition, de transformation et d'exploitation frauduleuse d'éléments identificateurs comme des «crimes liés à l'identité». Cependant, l'adoption généralisée par la presse écrite et les médias électroniques du terme «vol d'identité» paraît aujourd'hui irréversible.

\section{Des statistiques à la fiabilité fragile}

Le discours médiatique sur le «vol d'identité» est essentiellement alimenté par des statistiques dont la fiabilité mérite elle aussi d'être attentivement examinée. En effet, devant le peu de statistiques officielles disponibles, la mesure du phénomène est principalement assurée par des intérêts privés. Le désintérêt relatif des autorités gouvernementales s'explique en partie par l'absence d'une incrimination pénale qui s'accompagne généralement (mais pas systématiquement) de la mise à jour des formulaires de recueil de la statistique policière et judiciaire (Pontell 
et al., 2008), mais également par la réticence à voir émerger une forme de délinquance à très fort volume contre laquelle peu de ressources répressives peuvent effectivement être mobilisées. Par ailleurs, les quelques études financées sur des budgets publics (Baum, 2006; Dupont, 2008; Sproule et Archer, 2008) sont rarement reconduites, ce qui empêche d'analyser l'évolution du phénomène dans le temps.

L'espace statistique laissé vacant est donc occupé par des entreprises privées qui commanditent des sondages dont la consultation est payante, ou qui sont utilisés comme argument de commercialisation pour certains produits et services liés à la protection de l'identité. Ainsi, la version complète des résultats du sondage de l'entreprise Javelin Strategy and Research est offerte à la vente pour 3000 dollars $^{1}$, en plus d'être en partie financée par des acteurs du secteur bancaire comme Fiserv ou Wells Fargo. La première version de ce sondage annuel date de 2003, et il s'agit par conséquent de l'instrument de mesure du «vol d'identité» le plus fréquemment cité dans les médias. Dans ce contexte, le déclin du nombre de victimes observé par Javelin entre 2003 et 2007 prend un sens particulier qui ne doit pas déplaire aux commanditaires corporatifs du sondage. Au Québec, le sondage rendu public en mars 2008 par Sigma Assistel, une filiale de la compagnie d'assurances Desjardins Sécurité financière, insistait sur les conséquences négatives du «vol d'identité», comme les restrictions de l'accès au crédit ou encore le stress induit par les démarches de restauration de l'identité. Le fait que Sigma Assistel offre un service «assistance vol d'identité» n'est évidemment pas le fruit du hasard. En France, le premier sondage sur l'usurpation d'identité (le terme privilégié dans l'hexagone) publié en 2009 fut quant à lui sponsorisé par Fellowes, une entreprise américaine qui fabrique et distribue des destructeurs (ou déchiqueteurs) de documents ${ }^{2}$.

Les problèmes associés aux sondages sur le «vol d'identité» ne concernent pas seulement les biais inhérents à ceux qui en financent la réalisation et la diffusion. Ils se confondent également avec les ambiguïtés terminologiques décrites plus haut. En effet, en l'absence d'une définition claire et connue de tous, les répondants n'associent pas nécessairement certaines fraudes dont ils ont été victimes à un «vol

1. www.javelinstrategy.com/Brochure-170\#DownloadReport, consulté le 10 avril 2010.

2. www.credoc.fr/pdf/Sou/synthese \% 20Presentation \% 20fellowes \% 20- \% 20CREDOC $\%$ 202009\% 20\% 5BLecture \% 20seule \% 5D.pdf, consulté le 16 novembre 2010. 
d'identité». À titre d'exemple, dans le sondage mené au Québec (Dupont, 2008), seulement 2,5\% des personnes interrogées affirmaient avoir été victimes d'un «vol d'identité» (qui leur avait été présenté comme une «fraude consistant à collecter et à utiliser des renseignements personnels à l'insu et sans l'autorisation de la victime, et ce, à des fins généralement criminelles») au cours des douze mois précédents, alors que quatre vignettes correspondant aux principales techniques employées par les délinquants (utilisation frauduleuse d'une carte de paiement, utilisation d'éléments identificateurs pour obtenir des facilités de crédit, accès frauduleux à des comptes bancaires en ligne, et obtention frauduleuse de services non financiers) laissaient plutôt penser que le chiffre réel était de 5,7\%, soit un peu plus du double du pourcentage

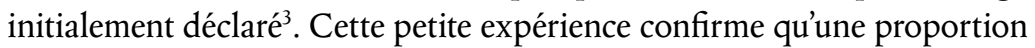
significative des victimes est ainsi absente des sondages qui tiennent pour acquis que le terme «vol d'identité» est rentré dans l'usage courant et qu'une signification précise lui est associée au sein de la population.

\section{Le délinquant inconnu}

Si les sondages se succèdent à intervalles réguliers afin d'établir le nombre et le profil des victimes, ainsi que les risques et les préjudices auxquels ces dernières sont exposées, les délinquants sont au contraire presque totalement délaissés par les chercheurs. Cela fait dire à Marron (2008: 34) que ce crime «sans cause» se voit doté d'une existence autonome qui ne s'encombre pas de considérations susceptibles d'en atténuer la portée allégorique. Les experts du secteur privé ne manquent pas en effet d'utiliser le levier que constitue ce manque de données pour imposer leur propre représentation des voleurs d'identité: appartenant à des réseaux structurés - quand ils ne sont pas rattachés au redoutable crime organisé -, ces super-délinquants opéreraient à l'échelle mondiale et mobiliseraient des compétences techniques hors normes pour se procurer des millions d'identités qui seraient ensuite revendues sur des marchés clandestins où les numéros de cartes de crédit et les codes d'accès aux comptes bancaires s'échangeraient à des cours déterminés par les lois de l'offre et de la demande (voir à titre d'exemple Berinato, 2007; Symantec, 2008; Thibodeau, 2008).

3. Cet écart est également problématique pour les campagnes de prévention du «vol d'identité», puisqu'il laisse entendre qu'une proportion importante de la population ne s'estime pas concernée, alors même qu'elle y est objectivement exposée. 
Bien que quelques arrestations fortement médiatisées ${ }^{4}$ viennent périodiquement renforcer ce mythe du super-utilisateur (Ohm, 2008), la majorité des délinquants impliqués dans des «vols d'identité» et interpellés par les forces de l'ordre exhibe un profil beaucoup plus quelconque (Allison et al., 2005; Copes et Vieraitis, 2007; Gordon et al., 2007). Une analyse des caractéristiques de 867 accusés réalisée à partir d'informations publiées dans les médias américains et canadiens entre janvier 2008 et décembre 2009 démontre que les modes opératoires privilégiés sont de nature très rudimentaire. En effet, les délinquants agissent seuls dans $72,7 \%$ des cas, et leurs techniques d'acquisition des éléments identificateurs ne reposent pas sur la mobilisation d'une expertise technique très développée, puisque près de la moitié des cas $(46,3 \%)$ implique le vol physique de portefeuilles et de sacs à main, alors que l'utilisation frauduleuse de fichiers informatiques auxquels les fraudeurs ont accès dans le cadre de leur emploi légitime représente $29,9 \%$ des affaires. Les techniques de piratage abondamment décrites dans la presse, comme l'hameçonnage (le «phishing») ou le déploiement de botnets, n'ont été utilisées que par $6,9 \%$ des individus arrêtés. Internet n'est pas plus présent à l'étape de l'exploitation frauduleuse, puisque les deux techniques privilégiées par les délinquants pour extraire un gain des éléments identificateurs obtenus sont l'achat en magasin à l'aide de cartes de paiement volées ou contrefaites $(41,7 \%)$ et le retrait d'espèces dans des agences bancaires $(20,2 \%)$. Cette simplicité dans les moyens utilisés n'empêche pas de réaliser des profits substantiels, la médiane des montants obtenus par les individus arrêtés s'élevant à 26793 dollars, ce qui procure à cette activité un rendement très favorable.

Ainsi, loin d'être l'apanage d'une nouvelle catégorie de criminels internationaux dotés de compétences singulières, le «vol d'identité» permet plutôt à de petits délinquants opportunistes de se diversifier vers des activités peu risquées et très profitables.

\section{La confiance des consommateurs}

La forte proportion de la population victime chaque année d'un «vol d'identité» s'explique donc en partie, si l'on en juge par les résultats de la section précédente, par la relative facilité avec laquelle les éléments

4. Comme celle d'Albert Gonzalez, responsable du vol de plusieurs dizaines de millions de numéros de cartes de crédit (Stone, 11 août 2008). 
identificateurs peuvent être obtenus et frauduleusement convertis en profits attrayants par des délinquants sans compétences particulières. Dans un tel contexte, il serait logique de penser que la confiance de la population dans les institutions bancaires, particulièrement exposées à ce type de fraude, connaîtrait un déclin marqué. Pourtant, les résultats du sondage mené au Québec (Dupont, 2008: 23) contredisent cette intuition: les banques figurent au contraire en tête du palmarès des institutions dans lesquelles les citoyens placent leur confiance afin de les protéger contre le «vol d'identité» et la cybercriminalité $(68,5 \%$ de confiance), devant la police $(66,5 \%)$ et les organismes de protection des consommateurs $(66 \%)$.

Ce paradoxe apparent représente l'une des clés de l'interprétation du «vol d'identité», non plus seulement comme un phénomène purement criminologique pouvant s'expliquer mécaniquement par la convergence de cibles attractives, de délinquants motivés et l'absence de gardiens compétents, mais aussi comme la conséquence de transformations économiques et sociales beaucoup plus profondes ayant durablement modifié la structure des opportunités criminelles (Cohen et Felson, 1979: 593).

\section{La coévolution du vol d'identité et des systèmes de paiement}

Dans la mesure où plus des deux tiers des cas de «vol d'identité» impliquent l'utilisation frauduleuse de cartes de crédit ou de débit (Dupont, 2008; Pontell et al., 2008), une analyse de l'histoire et des caractéristiques de ce moyen de paiement devenu omniprésent dans nos vies quotidiennes est indispensable afin d'identifier les opportunités criminelles qui ont accompagné son développement, ainsi que celles dont il a causé l'extinction. Un système de paiement est généralement défini comme «l'assemblage de dispositions juridiques, de contrats et de solutions technologiques permettant des transferts de valeurs entre plusieurs parties dans le but d'atteindre les objectifs spécifiques de ces dernières» (Morriss et Korosec, 2005 : 19). L’histoire de la dématérialisation des systèmes de paiement commence dès le viI siècle avant notre ère, avec l'invention par les Lydiens des pièces de monnaie métalliques qui permettent l'émergence d'une unité de compte et la constitution d'un système d'échange de valeur liquide. Le développement des billets de change au XII ${ }^{e}$ siècle dans le nord de l'Italie permet 
la mise en place d'un réseau bancaire qui facilite l'accès des entrepreneurs au crédit. Bien qu'on retrouve la trace des premiers billets de banque en Chine à la fin du viI siècle (Ferguson, 2008: 28), l'Europe ne procède à cette troisième innovation décisive qu'au Xvir ${ }^{e}$ siècle. Celle-ci traduit une promesse de rédemption de l'émetteur à son porteur tout en retirant de la circulation une quantité importante de monnaie métallique (Evans et Schmalensee, 2005). En Amérique du Nord, c'est en 1690 qu'on voit apparaitre le papier monnaie dans le Massachusetts, afin de payer les efforts de guerre pour capturer la forteresse de Québec. L'informatisation progressive du système bancaire au début de la seconde moitié $\mathrm{du} \mathrm{xx}^{\mathrm{e}}$ siècle marque l'aboutissement du processus de dématérialisation de l'argent, puisque les flux financiers abandonnent le support de l'atome et prennent la forme de transferts d'électrons. Si ces quatre grandes évolutions furent avant tout guidées par la volonté de fluidifier les échanges économiques, chacune d'entre elles se vit confrontée à des risques criminels spécifiques qui suscitèrent en réaction des adaptations en matière de sécurité.

\section{Le développement des cartes de paiement}

L'idée de la carte de crédit est généralement attribuée à l'homme d'affaires Frank McNamara, qui l'aurait eue en 1950 à la suite d'un repas abondamment arrosé avec des clients dans un restaurant newyorkais à la mode. Lorsque l'addition lui fut présentée, il fut humilié de constater qu'il ne disposait pas de suffisamment d'espèces sur lui et dut demander à son épouse (qui n'avait pas été conviée à ces libations) de lui apporter en urgence le montant manquant. Afin d'éviter que cette fâcheuse situation ne se reproduise, et flairant une occasion d'affaire inexploitée, il fonda la même année le Diners Club, dont l'objectif était de permettre aux membres de régler leurs factures de restaurant en un seul paiement mensuel (Chutkow, 2001 : 59). Pourtant, l'auteur étatsunien Edward Bellamy avait publié dès 1888 un roman d'anticipation se déroulant en l'an 2000 dans une société égalitaire où l'argent aurait disparu et aurait été remplacé par des cartes de crédit qui permettraient de répondre à tous les besoins matériels de la population (Drury et Ferrier, 1984: 4). Dès le début du $\mathrm{xx}^{\mathrm{e}}$ siècle, des chaînes de stations-services et de nombreux grands magasins étatsuniens et canadiens offraient à leurs meilleurs clients des cartes privatives métalliques ou cartonnées qui leur procuraient des facilités de paiement (Biegelman, 2009: 16; Banque 
du Canada, 2008: 90). Toutefois, c'est l'avènement de la société de consommation et du tourisme de masse dans la période d'euphorie économique qui suivit la Deuxième Guerre mondiale qui permit véritablement l'essor de la carte de crédit comme moyen de paiement universel. À la suite du Diners Club, American Express lança une carte en 1958 (Grossman, 1987: 283), suivi la même année de BankAmericard qui prit le nom de Visa en 1976 (Chutkow, 2001). Au Canada, la carte BankAmericard fut introduite sous licence en 1968 par une coopérativecompétitive de quatre grandes banques (CIBC, Banque Royale, Toronto Dominion et Banque Nationale) sous le nom de Chargex (Chutkow, 2001). Les banques françaises avaient pour leur part commencé à offrir des cartes de crédit à leurs clients en 1967 sous le nom de Carte Bleue (Chabrier, 1968).

Si les cartes de crédit remportèrent au sein de la population un succès immédiat, leur viabilité financière s'avéra initialement problématique pour les banques émettrices qui firent face à des pertes colossales. En effet, le modèle commercial sur lequel repose la carte de crédit ne répond pas aux conditions de fonctionnement des marchés classiques. Les économistes utilisent le concept de «marché biface» pour expliquer son fonctionnement. La caractéristique principale d'un marché biface est que le vendeur doit satisfaire deux groupes distincts de clients, qui tirent un profit mutuel de leur mise en relation par l'intermédiaire du vendeur (Evans et Schmalensee, 2005 : 135). Dans le cas des cartes de crédit, les clients des banques émettrices sont les détenteurs de cartes et les commerçants. Les détenteurs de cartes bénéficient d'une facturation retardée des dépenses qui leur permet de financer un style de vie à gratification instantanée, d'une protection contre la perte et le vol à un niveau prédéfini, et peuvent également accéder à des systèmes de fidélisation qui leur donnent l'impression d'obtenir une meilleure qualité de service. Pour leur part, les commerçants sont protégés contre les défauts de paiement des consommateurs (chèques sans provision ou comptes en souffrance), transfèrent à une tierce partie le financement des ventes ne pouvant être payées comptant, allègent leurs tâches de comptabilité, réduisent leur exposition aux risques de vol et de malversation inhérents à la manipulation des espèces et des chèques, et peuvent accéder à des informations détaillées sur les détenteurs de cartes qui sont des clients fiables, puisque déjà approuvés par les institutions bancaires émettrices (Morris et Korosec, 2005 ; Arango et Taylor, 2008). 
Cependant, pour mettre un tel marché en place, une masse critique de détenteurs de cartes et de commerçants qui acceptent ces cartes doit être constituée de manière simultanée. En effet, il est difficile de convaincre les commerçants d'accepter les cartes (et les coûts afférents) si ces dernières ne sont utilisées que par un nombre limité de clients potentiels, et ceux-ci n'auront aucun intérêt à détenir un nouveau moyen de paiement si celui-ci n'est pas universellement accepté, comme l'est l'argent comptant. Afin de résoudre ce dilemme, les banques émettrices inondèrent donc le marché de cartes pré-approuvées qui furent distribuées sans vérification de solvabilité à l'ensemble de leurs clients existants. Cette approche commerciale agressive se traduisit par des pertes financières de plusieurs dizaines de millions de dollars causées par une accumulation de comptes débiteurs en souffrance, menaçant la survie de ce nouveau moyen de paiement (Grossman, 1987: 286; Chutkow, 2001).

\section{L'émergence de nouvelles opportunités criminelles}

Les fraudeurs comprirent également très rapidement la manne que pouvait représenter le lancement des cartes de crédit. La distribution par voie postale de nouveaux moyens de paiement, qui étaient activés dès leur émission, ouvrit la voie à des vols massifs de courrier, à la corruption des employés des postes, à la revente des cartes ainsi obtenues sur le marché noir ou à leur utilisation dans des commerces où des complices avaient été recrutés (Chabrier, 1968; Chutkow, 2001). Aux États-Unis, le Better Business Bureau estimait ainsi que pas moins de 300000 cartes avaient été volées en 1967, et évaluait à 1,2 million le nombre de cartes «perdues» qui faisaient néanmoins l'objet d'un usage occasionnel (Chabrier, 1968: 69), alors que d'autres statistiques laissaient entendre que le préjudice financier relié à la fraude à la carte de crédit se montait à environ 100 millions de dollars en 1969 (Bielgeman, 2009: 18). En l'absence de systèmes informatisés de gestion des transactions, les procédures de dépassement des plafonds de dépenses autorisées se faisaient par téléphone auprès d'une employée de la banque émettrice, ce qui pouvait prendre de longues minutes, autant dire une éternité dans un contexte marchand où l'objectif est de conclure la vente aussi rapidement que possible. Quant aux numéros de cartes volées ou en défaut de paiement, ils étaient distribués chaque mois aux commerçants sous la forme de «listes noires» qui devinrent rapidement aussi 
volumineuses que les annuaires du téléphone, ce qui en rendit la consultation rédhibitoire. Afin de lutter contre cette épidémie de fraude, plusieurs adaptations législatives et technologiques furent mises en œuvre.

Tout d'abord, le Congrès étatsunien vota en 1970 une loi prohibant l'envoi non sollicité de cartes de crédit afin d'éliminer les risques liés aux pratiques d'émission abusives des institutions financières. Ce resserrement de l'encadrement réglementaire aurait été impossible sans la pression des associations de consommateurs. Il illustre la responsabilité indirecte des banques dans la création de conditions favorables à la fraude, la rationalité de profit entrant en concurrence avec celle de sécurité dans un contexte économique très dynamique où l'acquisition de nouveaux clients est jugée prioritaire afin de conquérir de nouvelles parts de marché. En parallèle, des investissements considérables furent consentis par les deux grandes coopératives (Visa et Mastercard) qui se dotèrent de systèmes informatiques performants capables de détecter les tentatives de fraude et de les prévenir. Ainsi, le système BASE 1 de Visa, dont le déploiement en 1973 coûta trois millions de dollars, fit-il économiser aux banques 30 millions de dollars en pertes financières reliées à la fraude dès sa première année d'utilisation (Chutkow, 2001 : 158). L'année suivante, Mastercard activa ses propres systèmes BankNet et $I-N e t$ (Evans et Schmalensee, 2005: 74). L'introduction successive de la piste magnétique au dos de la carte, de codes de vérification (cvv1 et cvv2), d'hologrammes, d'un cryptogramme ou plus récemment d'un microprocesseur contenant des données cryptées (la puce) doit être interprétée dans la logique d'un marché biface. Dans un tel contexte, les mécanismes de prévention de la fraude destinés à protéger les détenteurs de cartes ne doivent pas faire peser sur les commerçants des contraintes excessives qui dilueraient la facilité d'utilisation qui caractérise ce moyen de paiement, à moins que les niveaux de fraude atteignent des seuils tels que l'équilibre global du marché en soit menacé. Ce type de risque devient endémique lorsque les banques émettrices relâchent leurs critères et leurs procédures d'accès au crédit, lorsqu'elles inventent et mettent en marché de nouveaux produits financiers facilement exploitables par les délinquants (Tremblay, 1986), ou lorsque les contre-mesures permettant de neutraliser les technologies de sécurité se diffusent rapidement parmi les fraudeurs.

Contrairement à ce que laisse entendre le discours dominant sur le «vol d'identité», ce type de fraude n'a rien de très nouveau ni de très 
mystérieux. Il a véritablement pris des proportions considérables dès la seconde moitié des années 1960, après l'introduction du nouveau système de paiement par carte de crédit. Par ailleurs, les opportunités criminelles qui découlent de cette innovation économique ne sont pas stables. Elles dépendent en grande partie des procédures et des technologies associées à l'émission et à l'utilisation des cartes, qui sont elles-mêmes partiellement déterminées par les niveaux de fraude, mais aussi et surtout par des exigences mercantiles qui viennent parfois éroder la sécurité des transactions. Dans cette perspective, les niveaux de délinquance sont en grande partie déterminés par les résultats de ces interactions réciproques entre le système de paiement par carte et les fraudeurs, qui en testent constamment les faiblesses.

L'utilisation parcimonieuse des stratégies de prévention par les émetteurs de cartes s'explique par la nécessité de préserver la fluidité des transactions et des échanges. Toutefois, cela ne signifie pas qu'ils restent passifs face aux risques d'abus qui menacent la légitimité du système de paiement, et par extension leurs profits financiers.

La mise en place de mécanismes privés de règlement des conflits

La réponse des trois grandes entreprises gestionnaires (Visa, Mastercard et American Express) doit plutôt être trouvée dans la mise en place d'un système très élaboré de résolution des conflits. En effet, la complexité du système de paiement mis en place au début des années 1950 se traduisit par la fragmentation des rôles et la multiplication des acteurs organisationnels qui participent à son fonctionnement. L'écosystème des paiements par carte se compose à ce titre des entreprises gestionnaires, des banques qui émettent les cartes, des entreprises qui fournissent des services aux émetteurs (notamment par le biais de dossiers de crédit et d'instruments d'évaluation des risques que représentent les détenteurs), des «acquéreurs» qui fournissent aux commerçants les équipements et les services requis pour traiter les paiements, des commerçants et des porteurs de cartes.

Comme nous l'avons vu dans la section précédente, cette complexité et la structure particulière du marché ainsi créé génèrent de nombreuses occasions de fraude et d'abus. Afin de résoudre promptement les conflits qui ne manquent pas de se manifester, des mécanismes privés de règlement ont été intégrés au système de paiement par les entreprises gestion- 
naires (Morriss et Korosec, 2005). Concrètement, les clients qui contestent une transaction disposent d'un recours auprès de l'institution ayant émis leur carte de crédit, et peuvent espérer obtenir un règlement de leur plainte dans un délai de quelques semaines - ou de quelques mois tout au plus pour les cas les plus épineux. Ce processus de règlement standardisé est caractérisé par des échanges d'information très structurés entre les parties qui permettent de détecter assez rapidement les acteurs qui exploitent le système dans une logique contraire à l'intérêt collectif. Ainsi, des commerçants qui font l'objet d'un nombre élevé de plaintes s'exposent-ils à un rehaussement des frais de gestion qu'ils doivent payer ou à un retrait de leur privilège d'accès au réseau, alors que les détenteurs qui accumulent un nombre déraisonnable de réclamations voient leur réputation entachée et peuvent s'exposer à l'augmentation des taux d'intérêt qui leur sont appliqués. Par ailleurs, le fait que les perdants assument les coûts de la transaction contestée constitue un puissant levier de contrôle des abus.

Un tel système a comme objectif implicite de limiter l'implication des institutions judiciaires dans les processus de résolution des conflits. Ses modalités de fonctionnement s'opposent d'ailleurs sur de nombreux points à celles qui caractérisent les affaires référées devant les tribunaux. On peut souligner par exemple le fait que les procédures sont conduites par des non-juristes, ce qui limite le recours au jargon technique ou l'impression d'asymétrie de l'expertise entre les parties et l'autorité adjudicatrice. Les procédures sont également conduites par téléphone ou par correspondance, ce qui améliore la disponibilité du système de résolution et n'entraîne pas l'obligation pour les parties de se déplacer et d'être toutes présentes au même moment et au même endroit. S'il viole de nombreux principes de justice procédurale, ce système de résolution se distingue néanmoins par son efficacité et sa rapidité, ainsi que par sa capacité d'adaptation aux comportements stratégiques détectés chez certaines parties. Ces modalités particulières de règlement des conflits expliquent en grande partie la satisfaction et la confiance manifestées par l'opinion publique envers les institutions financières, malgré un volume de fraude très élevé.

\section{L'extinction d'opportunités criminelles «traditionnelles»}

Ainsi, la croissance indéniable du «vol d'identité» ne peut être comprise qu'à travers la transformation des modes de consommation rendue 
possible par l'invention de la carte de paiement. La position hégémonique acquise par cette dernière vis-à-vis de systèmes de paiement plus anciens comme le papier monnaie ou les chèques explique en grande partie l'intérêt qu'y portent les délinquants et les fraudeurs. À mesure qu'une proportion croissante des transactions migre vers les cartes de paiement, on assiste à un transfert des opportunités criminelles qui exploitent les nombreuses faiblesses techniques et procédurales inhérentes à la dématérialisation des flux financiers. Au Canada, l'association Interac qui gère le système de paiement par débit direct estime ainsi que $76 \%$ des transactions commerciales effectuées en 2008 s'étaient réglées à l'aide de cartes de débit ou de crédit, les espèces ne concernant que $22 \%$ des achats. Par contraste, dix ans plus tôt (en 1997), les achats en espèces représentaient près de la moitié des règlements (Interac, 2009). La Banque du Canada arrive par des calculs différents aux mêmes résultats, et en conclut que les moyens de paiement électroniques sont en voie de remplacer de manière durable les billets dans les paiements de détail (Taylor, 2006: 30). La part des chèques est pour sa part négligeable en volume, mais elle reste significative en valeur ${ }^{5}$, notamment en raison de son utilisation quasi systématique pour payer certaines dépenses importantes de la vie quotidienne comme le loyer ou l'accès à certains services de base (électricité, téléphone, services publics, etc.). Il serait surprenant que les délinquants n'aient pas tiré les conséquences de cette évolution des habitudes de consommation et ne se soient pas adaptés à ce nouvel environnement.

La question que les criminologues doivent poser dans ce contexte n'est pas de savoir si le nombre de «vols d'identité» connaît bien une croissance fulgurante, ce qui en soi ne nous apprendra pas grand-chose, mais plutôt de confronter l'ampleur des fraudes constatées au volume des transactions financières réalisées par l'entremise des cartes de paiement. Ainsi, le montant des fraudes à la carte de crédit enregistré par l'Association des banquiers canadiens pour l'année 2009 pourrait sembler considérable avec des pertes de plus de 358 millions de dollars, mais ramené aux statistiques globales d'utilisation de ce moyen de paiement, il ne représentait plus que $0,13 \%$ des 264 milliards de dollars

5. Voir notamment les statistiques sur le volume et la valeur des effets papier et électroniques traités par les membres de l'Association canadienne des paiements depuis 1990 sur le site internet de cette dernière à l'adresse suivante: www.cdnpay.ca/publications/ acss_percent_fr.asp (consulté le 22 juin 2010). 
de transactions effectuées ${ }^{6}$. Par comparaison, le taux de perte net attribuable aux comptes en souffrance (défaut de paiement des factures au-delà d'une période de 90 jours) s'élevait pour la même période à $5,38 \%$ des soldes en cours. Le risque frauduleux semble par conséquent beaucoup mieux maîtrisé que le risque financier inhérent à la distribution du crédit à la consommation. Pour ce qui est des cartes de débit, le ratio des pertes frauduleuses est sensiblement identique, avec un pic en 2009 à $0,08 \%$ du montant des transactions réalisées ${ }^{7}$. On le voit bien, le «vol d'identité» est encore loin de remettre en question la viabilité du secteur bancaire, même si les gains réalisés par les fraudeurs sont loin d'être négligeables.

Par ailleurs, cette migration criminelle naturelle vers les moyens de paiement dominants s'accompagne d'externalités positives qui sont rarement prises en compte dans les analyses sur l'impact du «vol d'identité». En effet, le déclin de la monnaie en espèces et du chèque s'est traduit par le recul concomitant des formes de délinquance associées à la circulation de ces moyens de paiement. L'utilisation frauduleuse de chèques a été ainsi divisée par six entre le milieu des années 1980, où on observait un peu plus de 300 affaires par 100000 habitants, et le milieu des années 2000, où le taux était descendu à 50 affaires par 100000 habitants (Centre canadien de la statistique juridique, 2005: 9). Quant aux vols qualifiés dans les établissements commerciaux (incluant les banques et les commerces de détail), qui se caractérisent par le recours (ou la menace du recours) à une violence démesurée afin d'accéder rapidement à des quantités importantes d'espèces, ils ont connu une diminution de $34 \%$ au cours des dix dernières années, alors que ceux commis dans les lieux publics $(+8,3 \%)$ et dans les résidences $(+24,7 \%)$ augmentaient pendant la même période (Dauvergne, 2010 : 19). Une telle différence reflète certainement la plus faible disponibilité d'argent liquide dans les établissements commerciaux causée par le recours de plus en plus fréquent aux moyens de paiement électroniques.

6. Voir les statistiques sur la fraude par carte de crédit (2009) et les statistiques sur les cartes de crédit (2009) disponibles sur le site de l'Association des banquiers canadiens à l'adresse suivante: www.cba.ca/fr/component/content/publication/69-statistics (consulté le 22 juin 2010).

7. Selon les chiffres de l'association Interac à l'adresse suivante: www.interac.ca/media/ stats.php (consulté le 22 juin 2010). 
Si le «vol d'identité» semble donc bien profiter des occasions criminelles générées par l'apparition et l'adoption généralisée des cartes de crédit et de débit, la nouvelle écologie des moyens de paiement qui en découle a provoqué l'extinction d'opportunités criminelles classiques comme les fraudes par chèque et les vols qualifiés dans les établissements commerciaux.

\section{Conclusion}

À travers ces quelques éléments historiques et ces statistiques, j'ai souhaité démontrer dans quelle mesure il s'avère contre-productif de considérer le «vol d'identité» comme une forme de délinquance nouvelle et hors de contrôle. Il serait plus pertinent d'y voir la conséquence naturelle d'une évolution économique, technologique et institutionnelle engagée depuis une quarantaine d'années dans le secteur bancaire. Comme l'a montré Tremblay (1986: 244) dans un cadre plus restreint, la conception et le lancement de nouveaux produits financiers créent de nouvelles vulnérabilités qui sont rapidement détectées et exploitées par les délinquants, ce qui en retour conduit à des ajustements plus ou moins rapides des procédures de sécurité. De la même manière qu'il est impossible aux biologistes de comprendre l'évolution de certaines espèces animales sans s'intéresser à celle des plantes qui leur fournissent leur alimentation (Ehrlich et Raven, 1964), ou aux climatologues de modéliser le réchauffement de la planète sans intégrer dans leurs calculs la nature et l'ampleur des activités humaines, on imagine mal que les criminologues arrivent à comprendre la structure des crimes d'acquisition sans accorder une plus large place à l'étude des circuits économiques et financiers et des habitudes de consommation qui rendent possibles les transferts de richesses. Cette perspective coévolutionniste, qui s'intéresse aux processus impliquant des changements et des adaptations réciproques par deux groupes distincts d'acteurs (ou d'espèces) en interaction constante, qu'il s'agisse de compétiteurs ou au contraire de partenaires (Vermeij, 1994: 224), reste pourtant sous-développée en criminologie. Et lorsqu'elle est prise en compte, c'est souvent dans une perspective utilitariste de prévention situationnelle (Newman et Clarke, 2003) qui parvient rarement à se dégager du problème immédiat qui l'occupe. Pour en revenir au «vol d'identité», l'analyse que j'en propose ici met en lumière le rôle central joué par les quelques grands acteurs du système de paiement par carte que sont les institutions gestionnaires 
(Visa, Mastercard, American Express, ou encore Interac) et émettrices (les banques et autres établissements financiers). Il en résulte que l'État et ses organismes d'application de la loi ne peuvent prétendre jouer de rôle significatif dans la lutte contre le «vol d'identité» et sa prévention qu'à travers la mise en œuvre de mécanismes de collaboration ou de régulation dirigés vers ces acteurs, tout en gardant à l'esprit que ces derniers offrent avant tout à leurs clients un service dont l'utilité principale est la facilité d'usage. L'approche coévolutionniste permet ainsi de mettre en lumière l'existence de cette tension entre sécurité et utilité, qui sous-tend le développement des crimes liés à l'identité, et d'offrir une alternative plus crédible à la description a-historique et a-théorique actuellement dominante.

\section{Références}

Allison, S., Schuck, A., \& Lersch, K. M. (2005). Exploring the crime of identity theft: Prevalence, clearance rates, and victim/offender characteristics. Journal of Criminal Justice, 33 (1), 19-29.

Arango, C., \& Taylor, V. (2008). Coûts des divers modes de paiement: l'argent comptant est-il le moyen le moins onéreux pour les commerçants? Revue de la Banque du Canada, Hiver, 17-27.

Banque du Canada (2008). Si l'argent m'était compté. Ottawa: Banque du Canada.

Baum, K. (2006). Identity theft 2004 : First estimates from the National crime victimization survey. Washington DC: Bureau of Justice Statistics.

Berinato, S. (2007). Who's stealing your passwords? Global hackers create a new online crime economy. CIO.com: 17 septembre. Accessible en ligne à: www.cio.com/article/135500.

Biegelman, M. (2009). Identity theft handbook. Hoboken: John Wiley \& Sons.

Cavoukian, A. (1997). Vol d'identité: Qui se sert de votre no? Toronto: Commissaire à l'information et à la protection de la vie privée.

Centre canadien de la statistique juridique (2005). Une étude de faisabilité d'améliorer la mesure de la fraude au Canada. Ottawa: Statistique Canada.

Chabrier, P. (1968). Les cartes de crédit. Paris: Librairies Techniques.

Chutkow, P. (2001). Visa: The power of an idea. Chicago: Harcourt.

Cohen, S. (1972). Folk devils and moral panics: The creation of the Mods and Rockers. St Albans: Paladin.

Copes, H., \& Vieraitis, L. (2007). Identity theft: Assessing offenders' strategies and perceptions of risk. Birmingham: University of Alabama.

Drury, T., \& Ferrier, C. (1984). Credit cards. Londres: Butterworths.

Dupont, B. (2008). Résultats du premier sondage sur le vol d'identité et la cybercriminalité au Québec. Québec: Ministère de la Sécurité publique. 
Ehrlich, P., \& Raven, P. (1964). Butterflies and plants: A study in coevolution. Evolution, 18 (4), 586-608.

Evans, D., \& Schmalensee, R. (2005). Paying with plastic: The digital revolution in buying and borrowing. Cambridge: MIT Press.

Ferguson, N. (2008). The ascent of money: A financial history of the world. Londres: Penguin Books.

Finklea, K. (2009). Identity theft: Trends and issues. Washington DC: Congressional Research Service.

Gordon, G., Rebovitch, D., Choo, K. S., \& Gordon, J. (2007). Identity fraud trends and patterns: building a data-based foundation for proactive enforcement. Uttica College: Center for Identity Management and Information Protection.

Grossman, P. (1987). American Express: The unofficial bistory of the people who built the great financial empire. New York: Crown Publishers.

Interac (2009). Interac celebrates 25 years of connecting Canadians to their money. Toronto: Interac. Accessible en ligne à: www.interac.ca/media/press_25. php.

Jones, G., \& Levi, M. (2000). The value of identity and the need for authenticity. Londres: DTI Office of Science and Technology.

Koops, B.-J., Leenes, R., Meints, M., van der Meulen, N., \& Jaquet-Chiffelle, D.-O. (2009). A typology of identity-related crime. Information, Communication and Society, 12 (1), 1-24.

Marron, D. (2008). 'Alter reality' Governing the risk of identity theft. British Journal of Criminology, 48 (1), 20-38.

Monahan, T. (2009). Identity theft vulnerability: Neoliberal governance through crime construction. Theoretical criminology, 13 (2), 155-176.

Morriss, A., \& Korosec, J. (2005). Private dispute resolution in the card context: Structure, reputation and incentives. Journal of Law, Economics and Policy, 1, 393-472.

Newman, G., \& Clarke, R. (2003). Superbighway robbery: Preventing e-commerce crime. Cullompton: Willan.

Newman, G., \& McNally, M. (2005). Identity theft literature review. Washington DC: US Department of Justice.

Ohm, P. (2008). The myth of the superuser: Fear, risk and harm online. UC Davis Law Review, 41 (4), 1327-1402.

Pontell, H. (2009). Identity theft: bounded rationality, research and policy. Criminology or Public Policy, 8 (2), 263-270.

Pontell, H., Brown, G., \& Tosouni, A. (2008). Stolen identities : A victimsurvey. In M. McNally et G. Newman (Eds.), Perspectives on identity theft (57-85). Monsey: Criminal Justice Press.

Saunders, K., \& Zucker, B. (1999). Counteracting identity fraud in the information age: The Identity theft and assumption deterrence act. International Review of Law, Computers and Technology, 13 (2), 183-192.

Sproule, S., \& Archer, N. (2008). Measuring identity theft in Canada: 2008 consumer survey, MeRC working paper no. 23. Hamilton: McMaster University. 
Stone, B. (11 août 2008). Global trail of an online crime ring. The New York Times, A1.

Sutherland, E. (1940). White-collar criminality. American Sociological Review, 5 (1), 1-12.

Symantec (2008). Symantec report on the underground economy July 07 - June 08. Cupertino: Symantec.

Taylor, V. (2006). Tendances en matière de paiement de détail et résultats d'un sondage mené auprès du public. Revue de la Banque du Canada, Printemps, $27-40$.

Thibodeau, P. (2008). Credit card thieves ran a polite, professional help desk. Computerworld, 6 août. Accessible en ligne à:

www.networkworld.com/news/2008/080708-credit-card-thieves-ran-a.html.

Tremblay, P. (1986). Designing crime: The short life expectancy and the workings of a recent wave of credit card bank fraud. British Journal of Criminology, 26 (3), 234-253.

Vermeij, G. (1994). The evolutionary interaction among species: Selection, escalation, and coevolution. Annual Review of Ecology and Systematics, 25, 219-236.

ABSTRACT - In one of his classical articles, Edwin Sutherland highlighted as early as 1940 the mutual benefits that economists and criminologists could derive from a better integration of their knowledge. Although his interdisciplinary invitation was aimed at white collar crime, other forms of delinquency are particularly well suited to a similar approach, "identity theft" being among them. After having examined in the first section of this article the ambiguities and paradoxes that apply to contemporary analyses of "identity theft", I offer an alternative analytical framework that relies on the concept of coevolution between "identity theft» as we know it and the development of a new payment system from the late 1950s. By using historical and economics data, I show how this fundamental transformation in consumer habits contributed to the emergence of new criminal opportunities, the development of new security measures and private conflict resolution procedures, as well as the decline of other types of crimes.

KEYWORDS - Identity theft, payment systems, credit cards, coevolution, criminal opportunities, private conflict resolution procedures.

RESUMEN - En un artículo que se volvió un clásico de la criminología, Edwin Sutherland destacó en 1940 los beneficios recíprocos que podrían obtener los economistas y los criminólogos con una mejor integración de sus conocimientos. Si bien esta invitación se orientó principalmente al crimen de cuello blanco, otras formas de delincuencia se prestan bien a dicha interdisciplina, en primer lugar de las cuales figura el "robo de identidad". Luego de analizar en la primera parte las ambigüedades y paradojas que pesan en los análisis contemporáneos del "robo de identidad", en la segunda parte se propone un marco de análisis alternativo apoyado en el concepto de la coevolución, que vincula dicho robo, como lo conocemos ahora, con el desarrollo de nuevas formas 
de pago a partir del final de los años 1950. Con apoyo en datos históricos y económicos se ilustra cómo esta transformación fundamental de los hábitos de consumo ha contribuido al surgimiento de nuevas oportunidades criminales, al desarrollo de nuevos mecanismos de seguridad y procedimientos privados de solución de conflictos, así como a la disminución de otras formas de delincuencia.

PALABRAS CLAVE - Robo de identidad, medios de pago, tarjetas de crédito, coevolución, oportunidades criminales, procedimientos privados de solución de conflictos. 